\title{
Wavelet Analysis in Civil Engineering
}

\section{Pranesh Chatterjee}

Tata Steel, Netherlands

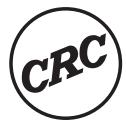

CRC Press

Taylor \& Francis Group

Boca Raton London New York

CRC Press is an imprint of the

Taylor \& Francis Group, an informa business 
MATLAB $^{\curvearrowleft}$ and Simulink ${ }^{\curvearrowleft}$ are trademarks of the MathWorks, Inc. and are used with permission. The MathWorks does not warrant the accuracy of the text or exercises in this book. This book's use or discussion of MATLAB ${ }^{\circ}$ and Simulink ${ }^{\circ}$ software or related products does not constitute endorsement or sponsorship by the MathWorks of a particular pedagogical approach or particular use of the MAT$\mathrm{LAB}^{\oplus}$ and Simulink ${ }^{\oplus}$ software.

\title{
CRC Press
}

Taylor \& Francis Group

6000 Broken Sound Parkway NW, Suite 300

Boca Raton, FL 33487-2742

(C) 2015 by Taylor \& Francis Group, LLC

CRC Press is an imprint of Taylor \& Francis Group, an Informa business

No claim to original U.S. Government works

Version Date: 20141209

International Standard Book Number-13: 978-1-4822-1056-9 (eBook - PDF)

This book contains information obtained from authentic and highly regarded sources. Reasonable efforts have been made to publish reliable data and information, but the author and publisher cannot assume responsibility for the validity of all materials or the consequences of their use. The authors and publishers have attempted to trace the copyright holders of all material reproduced in this publication and apologize to copyright holders if permission to publish in this form has not been obtained. If any copyright material has not been acknowledged please write and let us know so we may rectify in any future reprint.

Except as permitted under U.S. Copyright Law, no part of this book may be reprinted, reproduced, transmitted, or utilized in any form by any electronic, mechanical, or other means, now known or hereafter invented, including photocopying, microfilming, and recording, or in any information storage or retrieval system, without written permission from the publishers.

For permission to photocopy or use material electronically from this work, please access www.copyright.com (http://www.copyright.com/) or contact the Copyright Clearance Center, Inc. (CCC), 222 Rosewood Drive, Danvers, MA 01923, 978-750-8400. CCC is a not-for-profit organization that provides licenses and registration for a variety of users. For organizations that have been granted a photocopy license by the CCC, a separate system of payment has been arranged.

Trademark Notice: Product or corporate names may be trademarks or registered trademarks, and are used only for identification and explanation without intent to infringe.

\author{
Visit the Taylor \& Francis Web site at \\ http://www.taylorandfrancis.com
}

and the CRC Press Web site at

http://www.crcpress.com 


\section{Contents}

Preface

Acknowledgments

vii

About the Author

$i x$

Introduction

$x i$

xiii

1 Introduction to wavelets 1

1.1 History of wavelets 1

1.2 Fourier transform 2

1.3 Random vibration 5

1.4 Wavelet analysis 9

1.5 A brief review of wavelet properties 19

1.5.1 Continuous and discrete wavelet transform 20

1.5.2 Vanishing moments 22

1.5.3 Uncertainty principle 23

2 Vibration analysis of SDOF and MDOF systems in the wavelet domain

2.1 Wavelet-based discretization of ground motions 25

2.2 Time-frequency characteristics of wavelets 28

2.3 Formulation of SDOF system equation in wavelet domain 30

2.4 Wavelet basis function for ground motion process 32

2.5 Wavelet domain stochastic response of SDOF system 34

2.6 Statistical parameters and nonstationary peak responses 38

2.7 Wavelet domain stochastic response of MDOF system 41

3 Ground motion characterization and PSA response of SDOF system

3.1 Characterization of ground motions 49 
3.2 PSA response spectrum 52

3.3 Time history simulation by the Runge-Kutta fourth-order method 55

3.4 Fixed-base analysis of a tank 56

3.4.1 Basic assumptions 57

3.4.2 Equations of motion 57

3.4.3 Wavelet domain formulation 60

3.5 Numerical study 64

3.5.1 Ground motion characterization 64

3.5.2 Validation-PSA response 65

3.5.3 Validation - structural response 66

3.5.4 Wavelet analysis - structural response 69

4 Wavelet-based analysis of linear MDOF system

4.1 Description of the model 75

4.2 Equations of motion 78

4.3 Wavelet domain formulation of tankliquid-foundation system 81

4.4 Wavelet-based nonstationary system responses 90

4.5 Solution of transfer functions 95

4.6 Expected largest peak response 99

4.7 Numerical example 103

4.7.1 Impulsive response 104

4.7.2 MDOF analysis results 107

5 Wavelet-based nonstationary vibration analysis of a simple nonlinear system

5.1 Nonlinear system 111

5.2 Duffing oscillator 112

5.3 Perturbation method 116

5.4 Solution of the Duffing equation 117

5.5 Nonlinear system subjected to random vibration 118

6 Wavelet-based probabilistic analysis

6.1 Model and soil nonlinearity 133

6.2 General equations of motion 135

6.3 Equations based on yield conditions 137

6.4 Transfer functions 138

6.5 Response of the structure 143 
6.6 Probability evaluation 145

6.7 Validation and results 148

7 General applications

7.1 B-WIM NOR signal analysis 154

7.1.1 Bridge and vehicle model 155

7.1.2 Wavelet analysis of experimental NOR data 160

7.2 Stiffness degradation analysis 163

7.2.1 Description of the analytical model 164

7.2.2 Numerical approach to wavelet-based damage detection 167

7.2.3 Finite element model 167

7.2.4 Wavelet-based analysis of numerical results 171

7.3 Soil-structure-soil interaction analysis 173

7.3.1 Responses at tank base 178

7.3.2 Finite element model of the system 183

Appendix: MATLA $B^{\circledR}$ programmes 



\section{Preface}

When I started my doctoral research, my supervisor introduced me to the concept of wavelets. Initially, I was quite suspicious about the term wavelets. In time I came to learn that a wavelet is a powerful signal processing tool and can do some amazing things - extract the features hidden in a signal, for example. I became fond of wavelets and used the wavelet analytic technique for most of my research work. However, I realized that this particular topic was not easy to digest. More specifically, it was difficult to find suitable books outlining the application of wavelets in engineering. Since then I had in mind a latent desire to write such a book from my experience in this area that would be beneficial to interested postgraduate students and researchers. The opportunity came when I visited the stall of CRC Press at the 15th World Conference on Earthquake Engineering in 2012 in Lisbon, Portugal. I expressed my wish to write a book on applications of wavelets in civil engineering, and was approached soon thereafter when we all decided to go ahead.

I am greatly indebted to Professor Biswajit Basu and Professor Mira Mitra, who shared their valuable experiences with me and never hesitated to carry out in-depth discussions at times on the topic. I am grateful to my wife, Tanima, who has been a constant source of inspiration in my work, as well as to my two children, Sraman and Soham, who spent quality time with me to break up the monotony of writing. Last but not the least, sincere thanks are due to my friend Dr Debashish Bhattacharjee, who always encouraged me to remain strong in the face of daunting tasks.

Pranesh Chatterjee Delft, the Netherlands 
MATLAB $^{\circledR}$ is a registered trademark of The MathWorks, Inc. For product information, please contact:

The MathWorks, Inc.

3 Apple Hill Drive

Natick, MA 01760-2098 USA

Tel: 508-647-7000

Fax: 508-647-7001

E-mail: info@mathworks.com

Web: www.mathworks.com 


\section{Acknowledgments}

The author gratefully acknowledges the support of the Slovenian National Building and Civil Engineering Institute, ZAG, for provision of data from the SiWIM Bridge Weigh-in-Motion system (www.siwim.si) used in this book. 



\section{About the Author}

Pranesh Chatterjee, PhD, earned undergraduate and postgraduate degrees in civil engineering and a doctorate in engineering from Jadavpur University, India. His main research focus during his doctoral study was in the field of structural dynamics. During this research work he extensively used the wavelet-based analytical technique to formulate various problems in soil-structure-fluid interaction analyses. In later stages, $\mathrm{Dr}$ Chatterjee took up a postdoctoral fellowship in the Structural Mechanics section at Katholieke Universiteit te Leuven in Belgium and then was selected as a prestigious Pierse Newman Scholar at the University College Dublin in Ireland. After spending a considerable amount of time in academics and participating in a number of interesting research works that resulted in several journal and conference publications, he decided to move to industry. Since then he has worked in different fields and currently works as manager of the plasticity and tribology group of Tata Steel Europe in the Netherlands. Dr Chatterjee has always been active in research and its publication. 



\section{Introduction}

The main objective of Chapter 1 is to introduce to readers the concept and utility of wavelet transform. It begins with a brief history of wavelets referring to earlier works completed by renowned researchers, followed by an explanation of the Fourier transform. The chapter also shows the advantages of the wavelet transform over the Fourier transform through simple examples, and establishes the efficiency of the wavelet transform in signal processing and related areas. Chapter 2 first describes the discretization of ground motions using wavelet coefficients. Later, it explains the formulation of equations of motion for a single-degree-of-freedom system in the wavelet domain, and subsequently the same is used to build the formulation for multi-degree-of-freedom systems. The systems are assumed to behave in a linear fashion in this chapter. The wavelet domain formulation of equilibrium conditions of the systems and their solutions in terms of the expected largest peak responses form the basis of the technique of wavelet-based formulation for later chapters. Chapter 3 focuses on two distinct problems. The first is to explain how to characterize nonstationary ground motion using statistical functionals of wavelet coefficients of seismic accelerations. The second is to develop the formulation of a linear single-degree-of-freedom system based on the technique as described in Chapter 2 to obtain the pseudospectral acceleration response of the system. The relevant results are also presented at the end. Chapter 4 shows stepwise development of the formulation of a structure idealized as a linear multi-degree-of-freedom system in terms of wavelet coefficients. The formulation considers dynamic soil-structure interaction effects and also dynamic soil-fluid-structure interaction effects for specific cases. A number of interesting results are also presented at the end of the chapter, including a comparison between wavelet-based analysis and time history simulation. Chapter 5 describes the wavelet domain formulation of a nonlinear single-degree-of-freedom system. In this case, the nonlinearity is introduced into the system using a Duffing oscillator, and the solution is obtained through the perturbation method. Chapter 6 introduces the concept of probability in the wavelet-based theoretical formulation of a nonlinear two-degree-of-freedom system. The nonlinearity is considered 
through a bilinear hysteretic spring, and the probability conditions are introduced depending on the position of the spring with respect to its yield displacement condition. The analysis is supplemented with some numerical results. In the last chapter (Chapter 7), focus is on diverse applications to make readers aware of the use of wavelets in these areas. For this purpose, three different cases are discussed. The first one is related to the analysis of signals from bridge vibrations to identify axles of vehicles passing over the bridge. The second example explains the basic concept and formulation of stiffness degradation using a physical model. Thereafter, the chapter focuses on using a numerical technique to obtain the results of a degraded model (stiffness degradation through formation of cracks) and then compares the wavelet-based analysis of the results obtained from linear and nonlinear models. The third example is related to soil-structure-soil interaction. In this example, the wavelet analytic technique is used to obtain the results at the base of a structure considering dynamic soil-structure interaction. Subsequently, the forces, shears and moments thus obtained at the base of the model are applied at the supporting soil surface and a three-dimensional numerical model of this structure-soil interaction problem is used to obtain a nonstationary response within the soil domain. 


\section{Introduction to wavelets}

\section{I.I HISTORY OF WAVELETS}

In 1807, Joseph Fourier developed a method that could represent a signal with a series of coefficients based on an analysis function. The mathematical basis of Fourier transform led to the development of wavelet transform in later stages. Alfred Haar, in his PhD thesis in 1910 [1], was the first person to mention wavelets. The superiority of Haar basis function (varying on scale/frequency) to Fourier basis functions was found by Paul Levy in 1930. The area of wavelets has been extensively studied and developed from the 1970s. Jean Morlet, who was working as a geophysical engineer in an oil company, wanted to analyse a signal that had a lot of information in time as well as frequency. With the intention of having a good frequency resolution at low-frequency components, he could have used narrow-band short-time Fourier transform. On the other hand, in order to obtain good time resolution corresponding to highfrequency components, he could also have opted for broad-band shorttime Fourier transform. However, aiming for one meant losing the other, and Morlet did not want to lose any of this information. Morlet used a smooth Gaussian window (representing a cosine waveform) and chose to compress this window in time to get a higher-frequency component or spread it to capture a lower-frequency component. In fact, he shifted these functions in time to cover the whole time range of interest. Thus, his analysis consisted of two most important criteria - dilation (in frequency) and translation (in time) - which form the basis of wavelet transform. Morlet called his wavelets 'wavelets of constant shape', which later was changed by other researchers only to 'wavelets'. J.O. Stromberg [2] and later Yves Meyer [3] constructed orthonormal wavelet basis functions. Alex Grossmann and Jean Morlet in 1981 [4] derived the transformation method to decompose a signal into wavelet coefficients and reconstruct the original signal again. In 1986, Stephen Mallat and Yves Meyer developed multiresolution analysis using wavelets [3, 5, 6], which later in 1998 was used by Daubechies to construct her own family of wavelets. In 
1996, Daubechies [7] gave a nice, concise description of the development of wavelets starting from Morlet through Grossmann, Mallat, Meyer, Battle and Lemarié to Coifman, from the 1970s through mid-1990s. A pool of academicians, including pure mathematicians, engineers, theoretical and applied physicists, geophysical specialists and many others, have developed various kinds of wavelets to serve specific or general purposes as and when needed. Thus, though initiated mainly by the mathematicians, wavelets have gained immense popularity in all fields of applied sciences and engineering due to their unique time-frequency localization feature. It is due to this unique property that the wavelet transform has proved its ability (and reliability) in analysing nonstationary processes to reveal apparently hidden information that no other tool could provide. The application areas are wide, e.g. geophysics, astrophysics, image analysis, signal processing, telecommunication systems, speech processing, denoising, image compression and so forth. The wavelets have been applied analysing vibration signals. Some special techniques like discrete and fast wavelet transforms have been developed for this purpose. Before going into the discussion on wavelet analytic technique any further, it would be wise to review the basic theory on Fourier transform at this point.

\section{I.2 FOURIER TRANSFORM}

Most of the single-valued functions may be written as the summation of a series of harmonic functions within a desired range. This series is termed Fourier series. The concept of such a series has already been used by Daniel Bernoulli in connection to solving problems of string vibrations. However, it was Joseph Fourier, the French mathematician, who did a systematic study on Fourier series for the first time. Fourier series has found many applications in the fields of heat conduction, acoustics, vibration analysis, etc. The Fourier series for a function $f(x)$ in the interval $\alpha<x<\alpha+2 \pi$ is written as follows:

$$
f(x)=\frac{a_{0}}{2}+\sum_{n=1}^{\infty} a_{n} \cos (n x)+\sum_{n=1}^{\infty} b_{n} \sin (n x)
$$

where

$$
a_{0}=\frac{1}{\pi} \int_{\alpha}^{\alpha+2 \pi} f(x) d x
$$




$$
\begin{aligned}
& a_{n}=\frac{1}{\pi} \int_{\alpha}^{\alpha+2 \pi} f(x) \cos (n x) d x \\
& b_{n}=\frac{1}{\pi} \int_{\alpha}^{\alpha+2 \pi} f(x) \sin (n x) d x
\end{aligned}
$$

The readers should note that once the value of $\alpha$ is chosen as zero, the interval becomes $0<x<2 \pi$, and on choosing $\alpha=-\pi$, the range becomes $-\pi<x<\pi$. Thus, Fourier series can actually represent any periodic (and also nonperiodic) function as the sum of simple sine and cosine waves. This idea forms the basis of Fourier transform (FT), which is an extension of the Fourier series. In Fourier transform, the period of the function may extend to infinity. The Fourier transform retrieves the frequency content of a signal. It decomposes a signal into orthogonal trigonometric basis functions. The Fourier transform $\hat{X}(\omega)$ of a continuous function $x(t)$ is defined in the following equation:

$$
\hat{X}(\omega)=\frac{1}{\sqrt{2 \pi}} \int_{-\infty}^{\infty} x(t) e^{-i \omega t} d t
$$

In the above equation, the term $\hat{X}(\omega)$ gives the global frequency distribution of the time-dependent original signal $x(t)$. The original signal $x(t)$ can be further reconstructed using the inverse Fourier transform as defined below:

$$
x(t)=\frac{1}{\sqrt{2 \pi}} \int_{-\infty}^{\infty} \hat{X}(\omega) e^{i \omega t} d \omega
$$

The following Dirichlet conditions must be satisfied for Fourier transform and its reconstruction:

1. The time function $x(t)$ and its Fourier transform $\hat{X}(\omega)$ must be single-valued and piece-wise continuous.

2. The integral $\int_{-\infty}^{\infty}|x(t)| d t$ must exist that insists that if $|\omega| \rightarrow \infty$, $\hat{X}(\omega) \rightarrow \infty$.

3. The functions $x(t)$ and $\hat{X}(\omega)$ have upper and lower bounds (however, this is not a necessary condition). 
In case of signals obtained from experiments, they are discrete in nature as they are sampled at $N$ discrete time points with a sampling time of, say, $\Delta t$. These signals are analysed in the frequency domain using the concept of discrete Fourier transform (DFT), $\hat{X}_{D F T}(\omega)$, as defined below:

$$
\hat{X}_{D F T}\left(f_{n}\right)=\frac{1}{N} \sum_{n=0}^{N-1} x(n) e^{-i 2 \pi n t}
$$

It may be seen from Equation (1.7) that the DFT may be evaluated at discrete frequencies $f_{n}=\frac{n}{N t}$, where $n=0,1,2, \ldots, N-1$. The inverse DFT, as shown below, may be used to get back the original discrete time signal.

$$
x(n)=\frac{1}{t} \sum_{f_{n}=0}^{\frac{N-1}{N t}} \hat{X}_{D F T}\left(f_{n}\right) e^{i 2 \pi f_{n} n t}
$$

It may be noted here that the $N \Delta t$ in the equation above denotes the time length of the signal. The discrete Fourier transform computation requires evaluation of real and imaginary parts separately; thus, $2 \mathrm{~N}^{2}$ numbers of operations would be required. So, DFT works quite well when the signal length is short. If the signal becomes large with numerous discrete time points, DFT could become very tedious. The idea of fast Fourier transform (FFT) is developed, which is computationally more efficient in such cases because the FFT algorithm works on signals that must have as many samples as the power of 2 (i.e. $2^{m}$ samples). The FFT is much faster because it uses the results from previous computations and thereby reduces the number of operations required. It utilizes the periodicity and symmetry of trigonometric functions to compute the transform with approximately $N \log N$ numbers of operations.

If the time-dependent function $x(t)$ in Equation (1.6) has only one frequency, the corresponding frequency spectrum, $\hat{X}(\omega)$, is a Dirac delta function. So, if the frequency spectrum has only one frequency, say, $\hat{X}(\omega)=\delta\left(\omega-\omega_{0}\right)$, then on substituting $\hat{X}(\omega)$ in Equation (1.6) the following expression of $x(t)$ is obtained:

$$
x(t)=\frac{1}{\sqrt{2 \pi}} e^{-i \omega_{0} t}
$$


On substituting $x(t)$ on the right-hand side in Equation (1.5), the following equation is obtained, which is one of the definitions of the Dirac delta function.

$$
\hat{X}(\omega)=\frac{1}{2 \pi} \int_{-\infty}^{\infty} e^{i\left(\omega-\omega_{0}\right) t} d t=\delta\left(\omega-\omega_{0}\right)
$$

The readers should also know about Parseval's theorem and Parseval's identity at this stage, as these will be used in later chapters. Parseval's theorem is written as follows:

$$
\int_{-\infty}^{\infty}|x(t)|^{2} d t=\int_{-\infty}^{\infty}|\hat{X}(\omega)|^{2} d \omega
$$

This implies that the total energy content of a function $x(t)$ summed over all time $t$ is equal to the total energy contained in its Fourier transform summed across all of its frequency components.

$$
\frac{1}{\pi} \int_{-\pi}^{\pi}[f(t)]^{2} d t=\frac{a_{0}^{2}}{2}+\sum_{n=1}^{\infty} a_{n}^{2}+b_{n}^{2}
$$

The identity tells us that the sum of the squares of the Fourier coefficients of a certain function, say $f(t)$, is equal to the integral of the square of the function.

\section{I.3 RANDOM VIBRATION}

The random vibration is a nondeterministic motion that has a unique randomness in its characteristic. The vibrations induced in trains and road vehicles due to track and road surface roughness, wind excitations, ground motions and wave loading are common examples of random vibrations. Typically, a random vibration may be either a stationary or a nonstationary process. A common characteristic feature of these vibrations is that these are randomly varying in time, which obviously means that these are nondeterministic (and hence nonperiodic) in nature. This implies that in case of random vibrations, it is not be possible to predict the amplitude of vibration accurately at any specific instant; however, one may predict the probability of occurrence of acceleration or displacement amplitude at an instant. Unlike a pure sinusoidal vibration, a random vibration contains a continuous spectrum of frequencies. It may be worth mentioning here that the histogram of a random datum or 
signal tends to cluster near its mean value, and the histogram itself takes the shape of a bell-shaped curve, which is very similar to the Gaussian distribution curve. The peak values in a random vibration data do not maintain any fixed ratios with the root mean square (RMS) values. Let us assume that a sample function is generated every time a test is performed. This sample function may be different every time the test is performed. This creates an ensemble of sample functions that constitutes what we refer to as a random or stochastic process. For example, during ground shaking a sensor at a particular place records some data (precisely the movement of ground with respect to time at a given interval). For a different earthquake at the same site, the same sensor measures different data and thus records a new accelerogram. In this way, for an infinite number of measurements, an infinite number of data sets would be generated. This is called an ensemble of accelerograms. Any random process may be characterized by some statistical parameters like mean value, standard deviation, kurtosis, etc. If these properties remain constant with time, then vibration may be said to be a random stationary process. However, if the estimates of these statistical parameters vary with time, the vibration then represents a nonstationary random process for which instantaneous value of the datum or signal can never be predicted at any points of time.

In case of a stationary stochastic (or random) process, say $N(t)$, the power spectral density function (PSDF) comes in very handy. The PSDF $S(\omega)$ is defined as the Fourier transform of the corresponding correlation function $R(\tau)$ and is written as

$$
S(\omega)=\frac{1}{2 \pi} \int_{-\infty}^{\infty} R(\tau) e^{i \omega \tau} d \tau
$$

in which

$$
R(\tau)=\frac{1}{T-\tau} \int_{0}^{T-\tau} N(\tau) N(t+\tau) d t
$$

As $S(\omega)$ and $R(\tau)$ are even functions, their correlation function may be rewritten as

$$
R(\tau)=\int_{-\infty}^{\infty} S(\omega) \cos (\omega \tau) d \omega
$$


Putting $\tau=0$ in Equation (1.15), one may write the following:

$$
R(0)=E\left[N^{2}(t)\right] \int_{-\infty}^{\infty} S(\omega) d \omega
$$

The above equation readily tells us that the total mean square of the random stationary process, $N(t)$, is obtained by finding the total area under the power spectral density curve.

If a body is subjected to an excitation force that is a random process, its response must also be a random process. Let us concentrate on a single degree of freedom system in which a certain mass $m$ is connected to a spring (with stiffness coefficient $k$ ) and a dashpot (with coefficient, $c$ ) and the system is excited by an external time-varying force $f(t)$, which is an ensemble of several force-time histories. The system is shown in Figure 1.1, and the corresponding equation of motion is shown in Equation (1.16):

$$
m \ddot{x}(t)+c \dot{x}(t)+k x(t)=f(t)
$$

In the above equation, the dots represent differentiation with respect to time, and the terms $m$ and $x(t)$ denote the mass and the displacement response of the system, respectively. Now, as the input excitation $f(t)$ has been assumed to be a random process containing uncertainties, we should obtain the response $x(t)$ also as a random process. Let us assume that this input excitation $f(t)$ to the system represents an earthquake base excitation. For our case, we assume this to be the same as one of the ground motions recorded during the Loma Prieta earthquake (1989) at the Dumbarton Bridge site, shown in Figure 1.2. It may be noted that this excitation has a mean value of $0.0539 \mathrm{~mm} / \mathrm{s}^{2}$, and the corresponding histogram is shown in Figure 1.3.

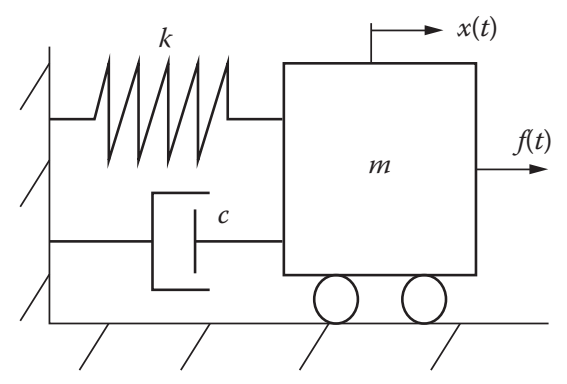

Figure I.I SDOF system subjected to external force. 


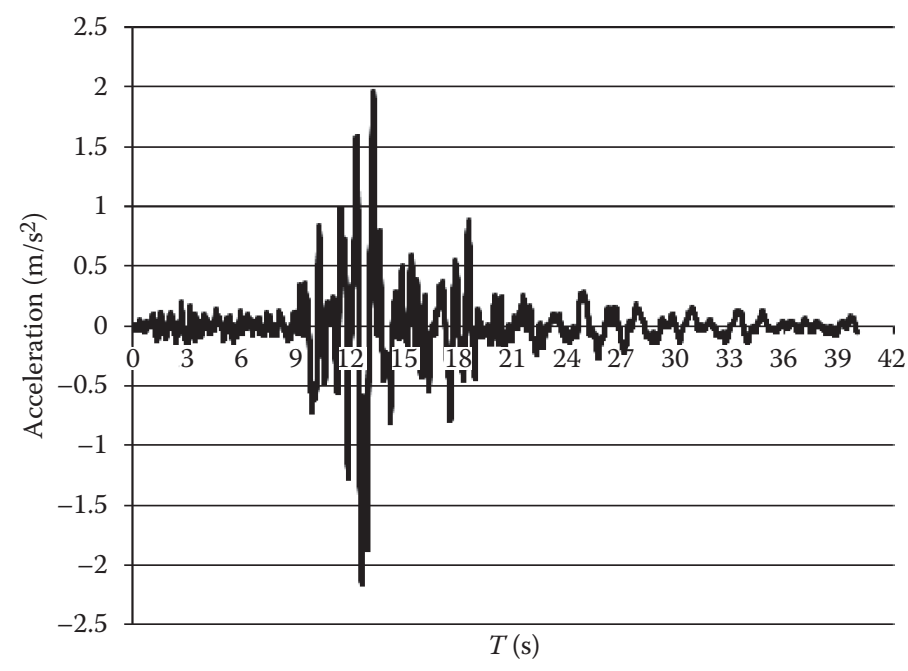

Figure I.2 Acceleration time history record during the Loma Prieta earthquake (1989) near the Dumbarton Bridge site (Coyote Hills).

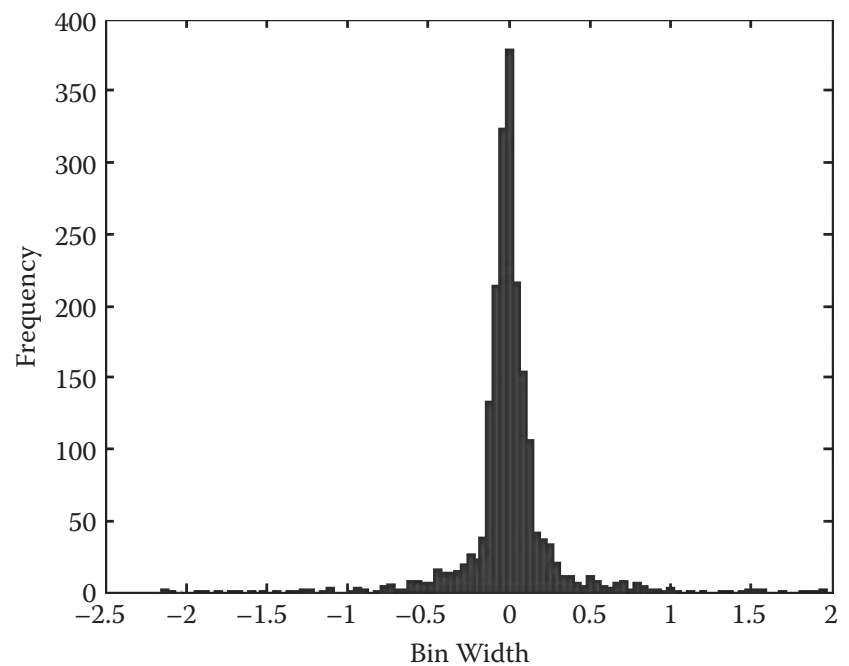

Figure I.3 Histogram of the Loma Prieta earthquake motion. 


\section{I.4 WAVELET ANALYSIS}

We have read in previous sections about Fourier transform and some important characteristics of random vibrations. The frequency spectrum gives in many cases thorough information about the sources of the signals that cannot be obtained from the time signal, e.g. the vibration level caused by rotating parts of a gearbox, the frequency content of vibration induced due to passage of high-speed trains underground, etc. Thus, a frequency analysis can be a valuable tool in locating the sources of vibrations as well as the level and frequency of vibrations. The Fourier transform, as discussed earlier, may work with periodic and nonperiodic functions, but these functions have to be stationary. The Fourier transform does not work with nonstationary functions or signals. However, it has become increasingly common in practice to analyse a nonstationary random signal or obtain responses of a structure when it is subjected to a nonstationary random excitation. The main purpose is to know the frequency content of a function at a certain time. The wavelet analytic tool has emerged as a powerful tool mainly due to its time-frequency localization property. Before going into the discussion on wavelets, it would be a good idea to understand the limitations of Fourier transform in analysis of signals that are nonstationary in nature.

Let us assume a stationary signal as shown in Figure 1.4. The signal has three frequency components, viz. 5, 10 and $25 \mathrm{~Hz}$. Thus, this signal has at any instant of time the presence of all three frequencies. On performing Fourier transform of this signal, we may identify the existing frequencies

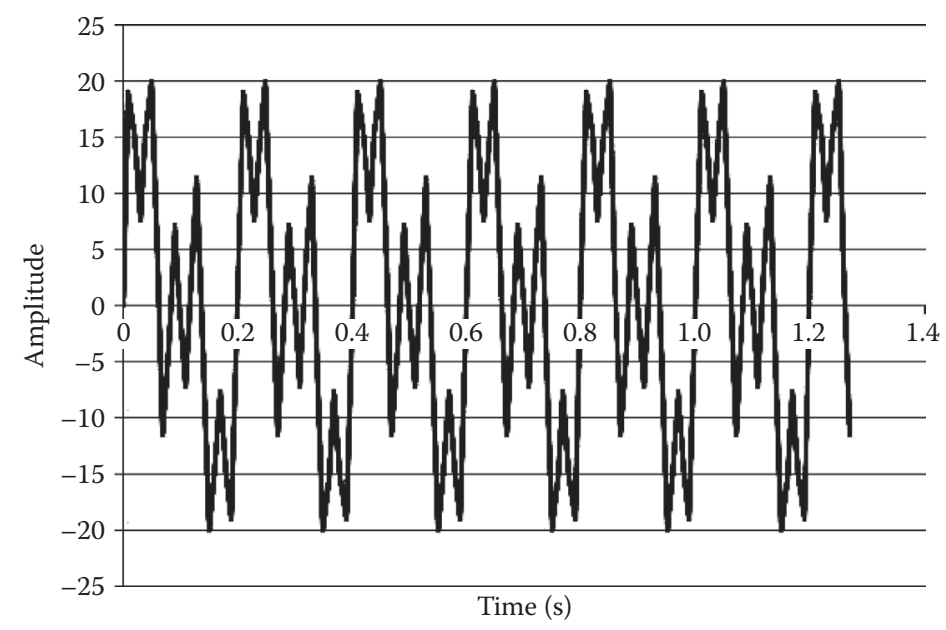

Figure I.4 Stationary signal consisting of frequencies 5, 10 and $25 \mathrm{~Hz}$. 


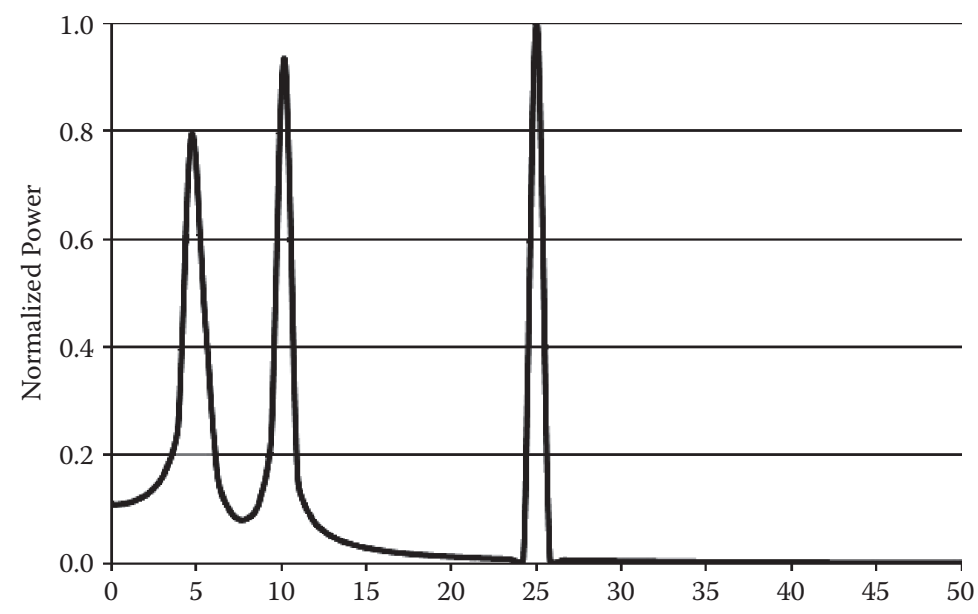

Figure I.5 Frequency spectrum of the stationary signal with frequencies 5,10 and $25 \mathrm{~Hz}$.

as shown in Figure 1.5. Thus, Fourier transform is proved to be efficient in revealing the frequency content of a signal.

Now, let us assume a nonstationary signal as shown in Figure 1.6. This signal shows the variation of the frequency of its amplitude in three distinct time zones. From 0 to $0.4 \mathrm{~s}$ it has a frequency of $25 \mathrm{~Hz}$, from 0.4 to $0.8 \mathrm{~s}$ the frequency is $5 \mathrm{~Hz}$, and for the remaining duration the frequency is $10 \mathrm{~Hz}$.

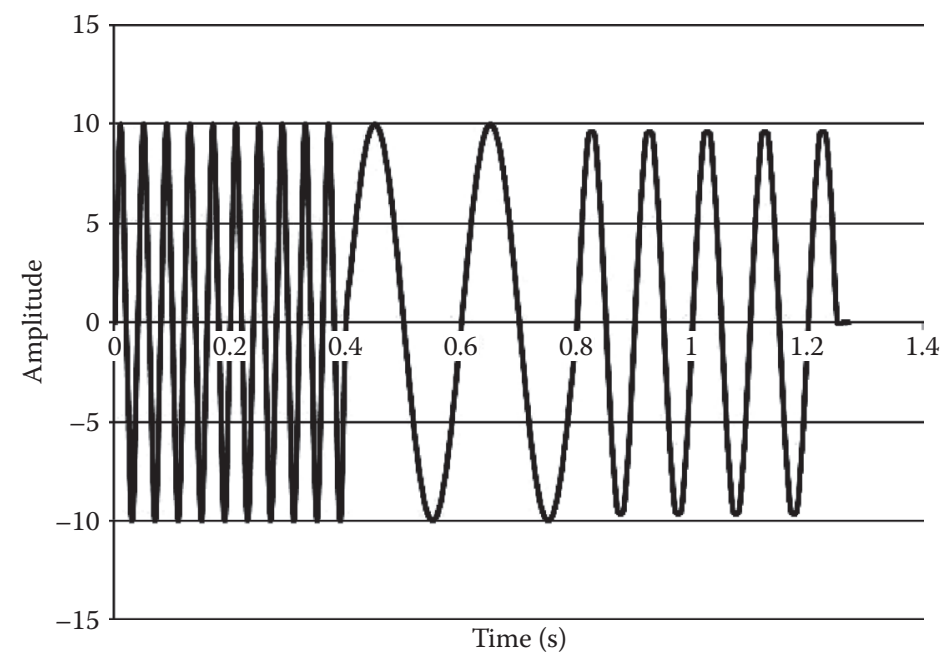

Figure I.6 Nonstationary signal with frequencies 25,5 and $10 \mathrm{~Hz}$. 


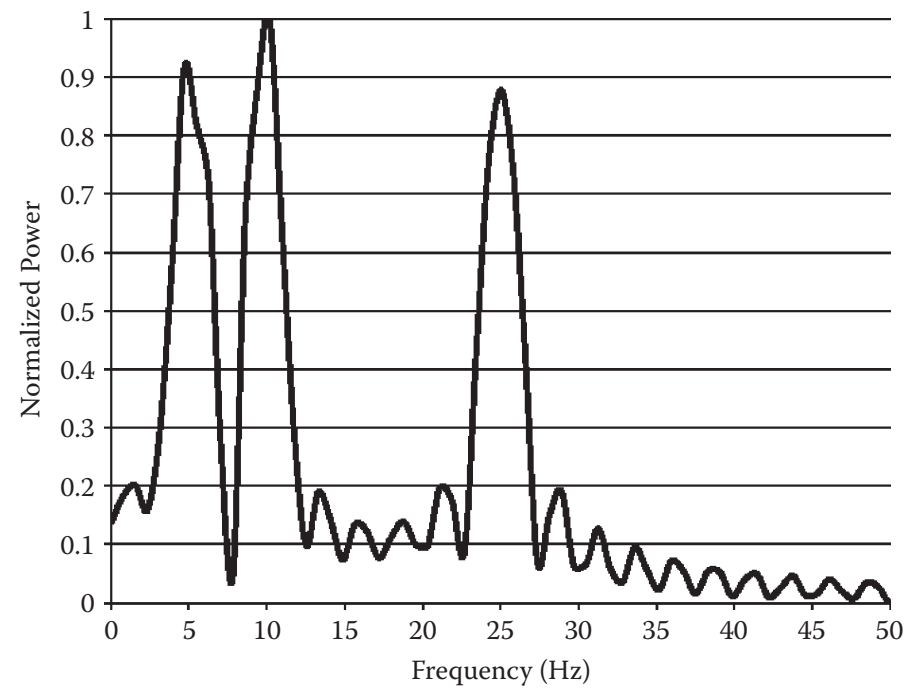

Figure I.7 Frequency spectrum of the nonstationary signal with frequencies 25,5 and $10 \mathrm{~Hz}$.

These frequencies can be easily identified on taking the Fourier transform of the signal. The corresponding Fourier transform of the signal is shown in Figure 1.7. Let us also assume another nonstationary signal as shown in Figure 1.8. On performing Fourier transform of this signal, we can clearly see in Figure 1.9 the dominant peaks again at frequencies 5, 10 and $25 \mathrm{~Hz}$.

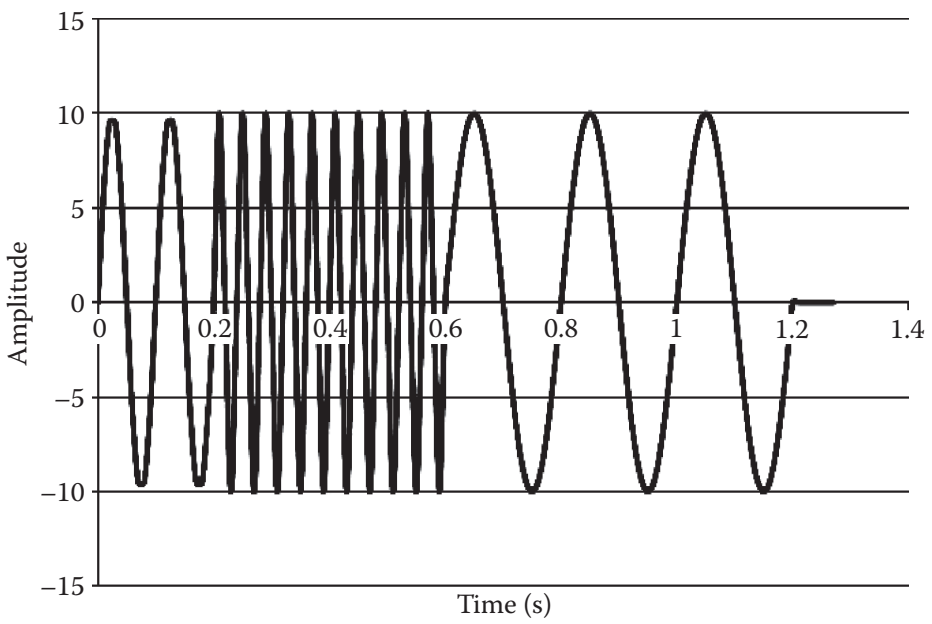

Figure I.8 Nonstationary signal with frequencies 10, 25 and $5 \mathrm{~Hz}$. 


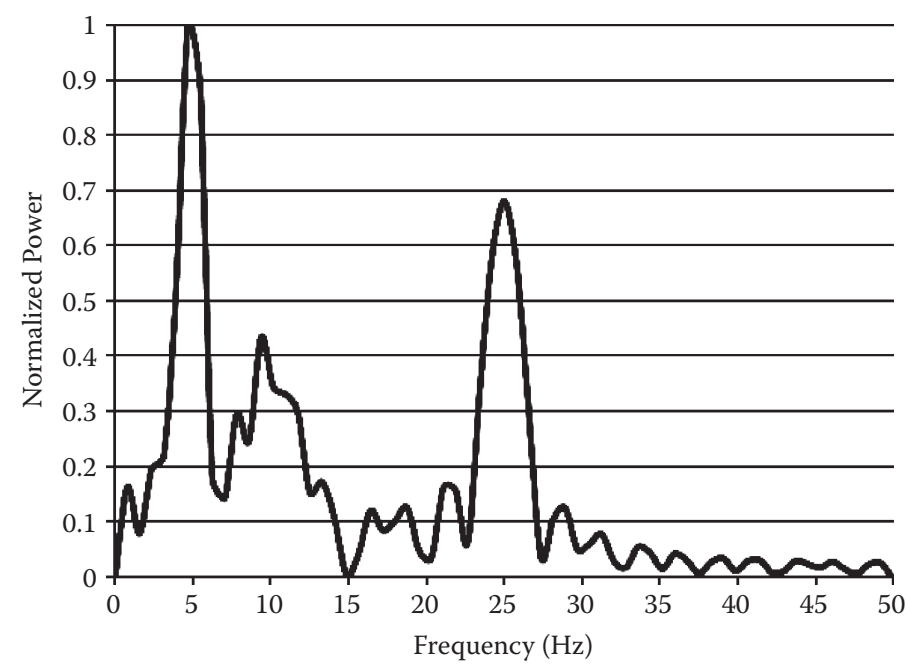

Figure 1.9 Frequency spectrum of the nonstationary signal with frequencies 10,25 and $5 \mathrm{~Hz}$.

Thus, we see that in all three signals, as shown in Figures 1.5, 1.7 and 1.9, the frequencies present are the same $-5,10$ and $25 \mathrm{~Hz}-$ but in different order. In the first case, the signal is stationary because the frequency content is the same at all instants of time. In the other two cases, though the frequency content is still the same, the occurrence of frequencies in the time domain is different. However, Fourier transformation of these nonstationary signals could not determine the order in which the frequencies appear in the time domain. This is a serious limitation of the Fourier transform in analysing stochastic nonstationary signals because in many physical and engineering problems we actually need to determine the presence of a frequency at a particular time instant. The Fourier transform clearly cannot handle this situation.

Thus, Fourier transform is not capable of extracting the information that we would like to know - time and frequency information - at the same time. Instead, what it does is generate the spectral density of the signal. It views the signal as a whole because it sums up all information over time; hence, the time information gets lost once a signal is Fourier transformed. Again, on taking inverse Fourier transform, one would integrate over the frequency range, and thus the frequency information is lost. So, the researchers tried to think in a more positive way and came up with an idea of short-time Fourier transform (STFT). This tool enabled the researchers to obtain the frequency content of a signal within a specified time interval. This could be achieved by breaking up the signal into several pieces and 
taking the Fourier transform of each piece. For this, a small window function, say $V(t)$, was necessary, which would help get the finite time variance and the finite frequency variance, respectively, as follows:

$$
\begin{aligned}
& t V(t) \in \mathcal{L}_{2}(\mathbb{R}) \\
& \frac{d V(t)}{d t} \in \mathcal{L}_{2}(\mathbb{R})
\end{aligned}
$$

In both Equations (1.17) and (1.18), $V(t) \in \mathcal{L}_{2}(\mathbb{R})$. So, the STFT of a function $x(t)$ with respect to the window $V(t)$ evaluated at translation $\tau_{0}$ and modulation $\Omega_{0}$ may be written as an inner or dot product as follows:

$$
\operatorname{STFT}(x(t), V(t))=\int_{-\infty}^{\infty} x(t) \overline{V\left(t-\tau_{0}\right)} e^{-i \Omega_{0} t} d t
$$

which may further be rewritten as

$$
\operatorname{STFT}(x(t) \cdot V(t))=\frac{1}{2 \pi} \int_{-\infty}^{\infty} \hat{x}\left(\overline{\hat{V}(-0)} e^{j\left(-\Omega_{0}\right) \tau_{0}} d\right.
$$

The window may be any suitable function like a Gaussian function, raised cosine function, triangular window, etc. as shown in Figures 1.10 to 1.12 . Thus, a properly translated window is actually used to multiply the function $x(t)$ to extract the information around $t=\tau_{0}$, and then the

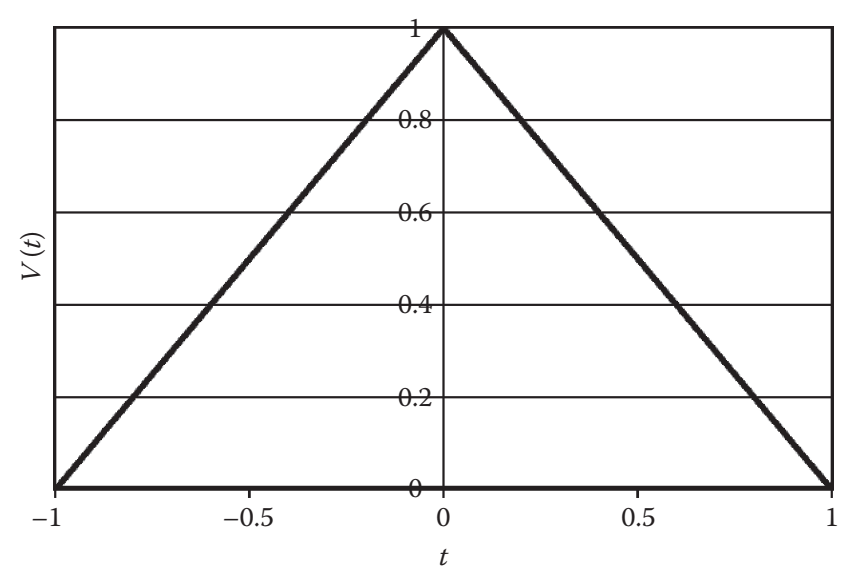

Figure I.10 Triangular window. 


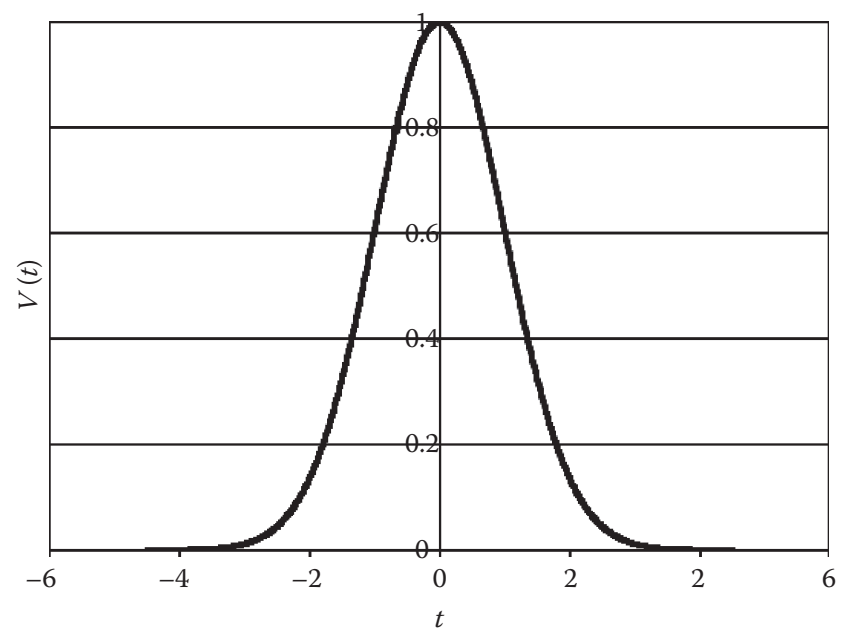

Figure I.II Gaussian window.

Fourier transform is taken; thus, it looks at the frequency content in a certain region. Equation (1.20) may actually be seen as Parseval's theorem, which says that the right-hand side of this equation is the dot product of the Fourier transforms of $x(t)$ and $\overline{V\left(t-\tau_{0}\right)} e^{-i \Omega_{0} t}$. However, even this STFT concept was not good enough to capture time-frequency information together. One can easily notice the main shortcoming of the STFT from Figures 1.10 to 1.12 , which is the limit in time and frequency. It

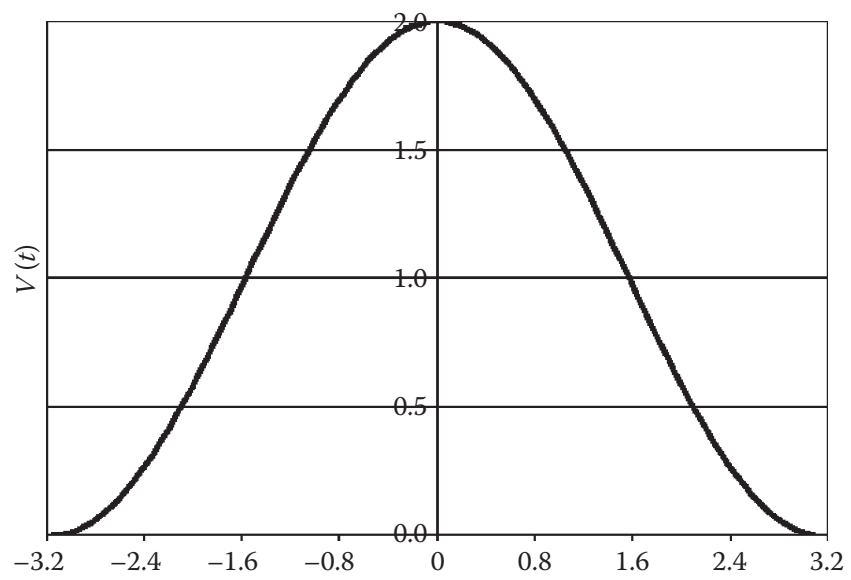

Figure I.I2 Raised cosine window. 
means that one may indeed choose a suitable window, but cannot vary the size of the window in the time-frequency plane. So, when one multiplies the signal by a chosen window function (with finite length), it covers only a part of the signal, unlike in FT, where it is done over the whole time range. Thus, the frequency resolution becomes poorer. In fact, in STFT, narrow windows produce good time resolution but poor frequency resolution, and it is the opposite for wider windows. This is actually the reiteration of Heisenberg's uncertainty principle, which states that it is impossible to measure the position and momentum of a particle simultaneously with infinite precision. In the present context, this may interpreted as a fact that it is not possible to obtain good resolutions in both time and frequency simultaneously with a fixed window size. To overcome this limitation, one must use windows of different sizes, thus leading to multiresolution analysis.

At this point, the concept of wavelet transform comes to the rescue. The wavelet transform overcomes the resolution problem faced by STFT. The continuous wavelet transform (CWT) is the dot (inner) product of the signal $x(t)$ and a wavelet $\Psi\left(\frac{t-\tau}{s}\right)$, where the wavelet $(\Psi)$ is translated by the time parameter $\tau$ in the numerator and dilated by the frequency parameter $s$ in the denominator. The term $\tau$ represents positive real numbers, and the term $s$ represents both positive and negative real numbers. The continuous wavelet transform of a function $x(t)$ with respect to the mother wavelet $\Psi$ evaluated at translation $\tau$ and scale $s$ is defined as

$$
\operatorname{CWT}_{x}(\tau, s)=\int_{-\infty}^{\infty} x(t) \frac{1}{\sqrt{s}} \frac{t-\tau}{s} d t
$$

The mother wavelet, $\left(\frac{t-\tau}{s}\right)$, in the above equation is a prototype from which the other window functions may be generated. All the wavelets are the dilated (scaling term is $s$, which is inverse of frequency) or compressed and shifted (translated in the time domain by an amount $\tau$ ) versions of the mother wavelet. The most important characteristic feature of wavelet transform over STFT is that the width of the multiplying window is changed as the transform is computed for each and every spectral component. The window function in a wavelet transform is an oscillatory function and is compactly supported having finite length. The scaling term $\sqrt{s}$ in the denominator of Equation (1.21) is used for the purpose of energy normalization so that a wavelet-transformed signal will have the same energy at all scales. Let us have a closer look at this equation and try to understand how a wavelet transform actually works. Let us also assume that we have a signal $x(t)$ known to us. First, a specific scale is chosen and the wavelet is placed at the beginning of the signal when the time count starts $(t=0)$. The signal is then multiplied 
by the wavelet function and subsequently integrated over all times and divided by the factor $\sqrt{s}$ to get a single value called the wavelet coefficient for a specific scale and at a particular time point. The operation is repeated with the same scale, but now the wavelet function is placed at a different time instant, which is basically a shift from the previous position by a fixed amount, $\tau$. This gives rise to the second wavelet coefficient. This goes on until one reaches the end of the signal. Thus, in doing so, one would ultimately obtain a row of wavelet coefficients for all time points but for a particular scale. Now, the scale is increased, and the whole process is repeated so as to obtain the second row of wavelet coefficients at all time points. In the end, when all desired values of scales are finished, one ends up with a complete array of wavelet coefficients for the signal $x(t)$. The product of the wavelet function with the signal at a particular location where the signal contains a spectral component generates larger amplitude; else the amplitude would be smaller or even zero.

Any function, say $y(t)$, may be expressed as a set of linear combinations of basis functions $\phi_{k}(t)$ with corresponding coefficients $v_{k}$ as follows:

$$
y(t)=\sum_{j} v_{k} \phi_{k}(t)
$$

Now, let us assume that $y(t)$ and $z(t)$ are two square integrable functions in the interval $[a, b]$ whose inner product is defined as

$$
\begin{aligned}
& \text { b } \\
& \langle y(t) z(t)\rangle \risingdotseq(t) . z(t) d t \\
& \text { a }
\end{aligned}
$$

The CWT as shown in Equation (1.21) is the inner product of the signal $x(t)$ with the basis function ${ }_{(\tau, s)}(t)$ where the $(\tau, s)(t)$ is written as

$$
{ }_{(\tau, s)}(t)=\frac{1}{\sqrt{s}} \frac{t-\tau}{s}
$$

The CWT depicts that the wavelet analysis is a measure of similar frequency content between the signal and its multiplier basis function. The wavelet coefficients generated from the calculation show the proximity of the signal to the wavelet basis function at the current scale. Thus, if there is a high correlation at a particular scale and time, the wavelet transform would generate a high value. When the wavelet is compressed, it represents high frequency. 
There are several families of wavelets developed by researchers in the past and present. Out of all these wavelets, only three are discussed here: Mexican hat wavelet, Morlet wavelet and Daubechies wavelet $(d b 2$, as an example). The first two types may be used to perform continuous wavelet transform, while the third one is suitable for discrete wavelet transforms. The Mexican hat wavelet, $\Psi_{\text {mexh }}$, is the second derivative of the Gaussian probability density function as defined below:

$$
\operatorname{mexh}=c e^{-\frac{x^{2}}{2}}\left(1-x^{2}\right)
$$

where $c=\frac{2}{\sqrt{3}(\pi)^{0.25}}$. The Morlet wavelet, $\Psi_{\text {mor }}$, has the following expression:

$$
\text { mor }=e^{-\frac{x^{2}}{2}} \cos 5 x
$$

The Mexican hat wavelet and Morlet wavelet are shown in Figures 1.13 and 1.14 , respectively.

When performing continuous wavelet transform of the nonstationary signals shown in Figure 1.6 (with frequencies in the order of 25, 5 and $10 \mathrm{~Hz}$ ) and Figure 1.8 (with frequencies in the order of 10, 25 and $5 \mathrm{~Hz}$ ) using Daubechies wavelet $d b 2$, one may obtain the two graphs (threedimensional plots) shown in Figures 1.15 and 1.16, which show the variation of wavelet coefficients with time.

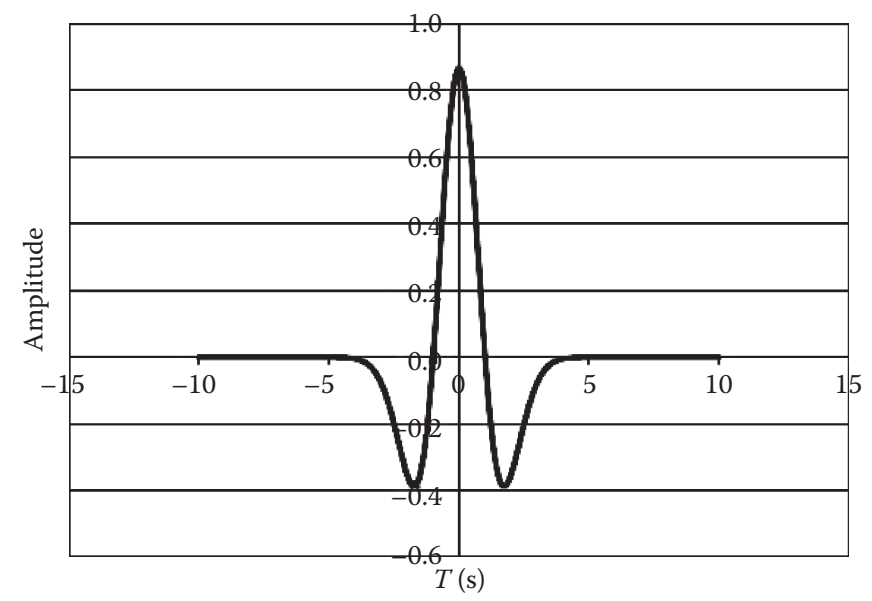

Figure I.13 Mexican hat wavelet. 


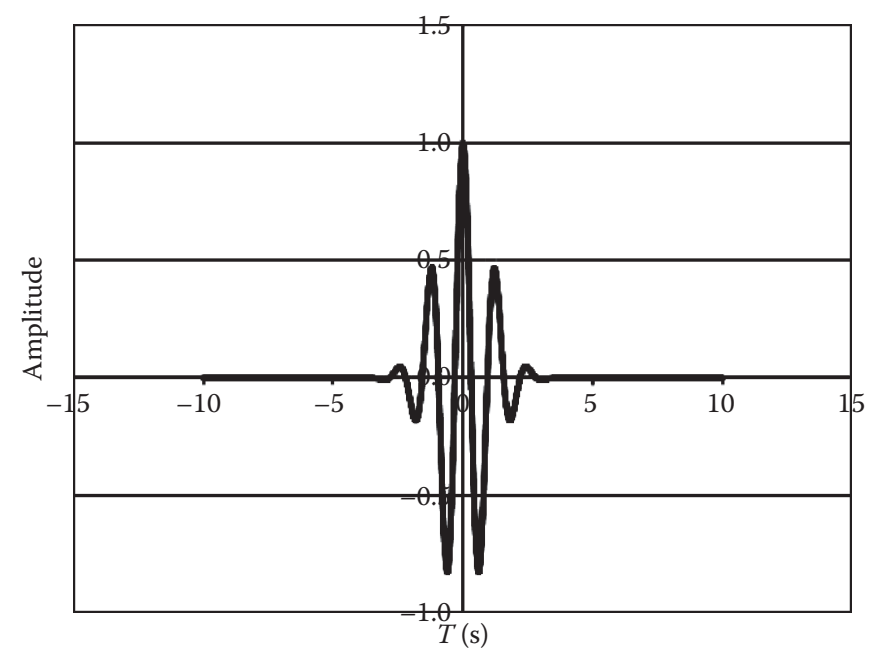

Figure I.I4 Morlet wavelet.

The two plots of wavelet coefficients of signals shown in Figures 1.15 and 1.16 clearly show the occurrence of frequencies at corresponding time regions. So, one can easily identify which frequencies are showing up at what frequencies. This is the most important feature of wavelet-based analysis. This unique time-frequency localization property has made the wavelets unique and most widely used for various purposes in the fields of signal processing, speech recognition, compression-decompression, image

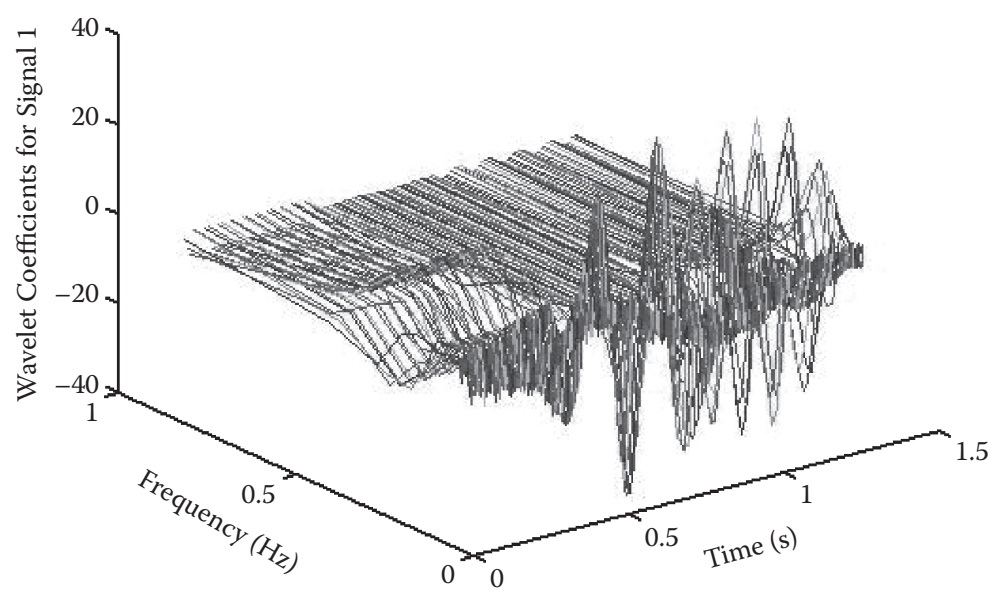

Figure I.15 Wavelet coefficients obtained from the signal as shown in Figure 1.6. 


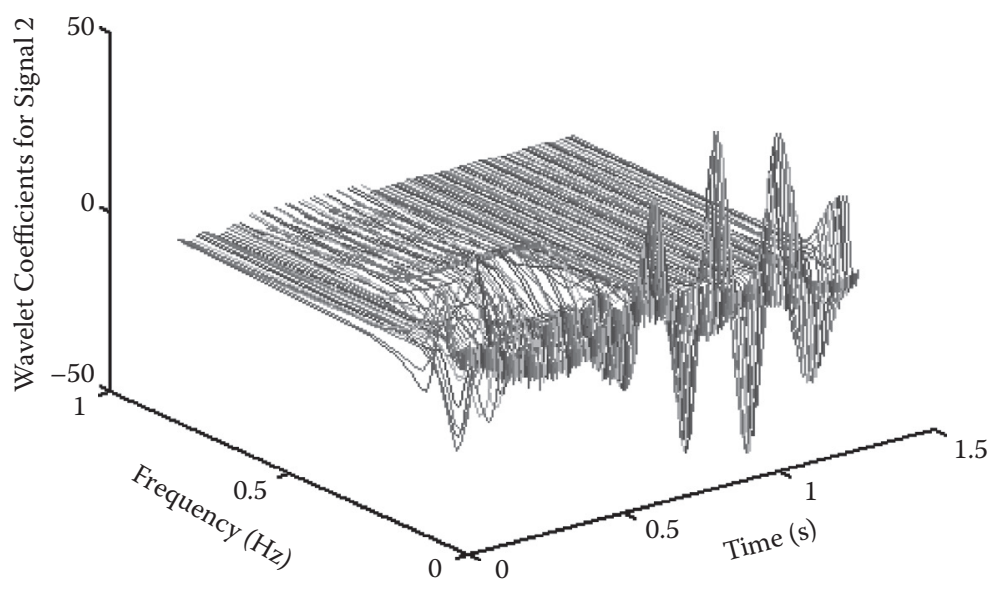

Figure I.16 Wavelet coefficients obtained from the signal as shown in Figure I.8.

processing, etc. In the next chapter we shall see how wavelets may be used to characterize ground motions and how the response of a single-degreeof-freedom system could be obtained performing the analysis in the wavelet domain.

\section{I.5 A BRIEF REVIEW OF WAVELET PROPERTIES}

In this section we are going to discuss some important properties of wavelet transform that will be used later in this book. The wavelet transform uses irregular shaped signals as basis functions that help in capturing discontinuities in the signal and abrupt changes. If a function $f(t)$ belongs to a space of all finite energy functions satisfying the following condition, the function can be decomposed by wavelet coefficients using wavelet transformation and later may be reconstructed from these coefficients using inverse wavelet transformation.

$$
\int_{-\infty}^{\infty}|f(t)|^{2} d t<\infty
$$

The choice of a wavelet function may be linked to the characteristics and properties of the signal. The discrete wavelet scheme is used for wavelet decomposition of a signal and its reconstruction. During this process, a signal is decomposed in high-scale (low-frequency) components called trends and low-scale (high-frequency) components called details. This 
decomposition is based on low- and high-pass filters, depending on the wavelet basis function used. At each stage (scale) of filtering, twice the amount of original data is generated, which is reduced by a factor of 2 (downsampling). The inverse process of upsampling and filtering is used during the reconstruction process using the low- and high-pass reconstruction filters. Besides wavelet function, there also exists a scaling function that is associated with the wavelet analysis of a signal. This scaling function is related to the trends of the wavelet decomposition. The scaling function is obtained by upsampling and convolving the low-pass with the high-pass reconstruction filter in an iterative manner. Daubechies [8] has given the following dilation equations for scaling $((t))$ and wavelet functions $((t))$.

$$
\begin{aligned}
(t) & =\sum c_{k}(2 t-k) \\
(t) & =\sum(-1)^{k} c_{1-k}(2 t-k)
\end{aligned}
$$

The uniqueness is defined as $\sum c_{k}=2$. The details about wavelet-based decomposition and reconstruction of signals may be found in the work by Grossman and Morlet [4].

\section{I.5.I Continuous and discrete wavelet transform}

The short-time Fourier transform (STFT) of a function $f(t)$ may be represented by the following equation, which is another form of Equation (1.19):

$$
F\left(\omega, t_{0}\right)=\int_{-\infty}^{\infty} f(t) e^{-i \omega t} g\left(t-t_{0}\right) d t
$$

Using this STFT definition, the wavelet function may be defined in the following manner. Let it be assumed that $g(t)$ is a window function that depends on time and frequency, and hence is defined as follows:

$$
g(t)=|\omega|^{\frac{1}{2}} g(\omega t)
$$

On substituting Equation (1.31) in Equation (1.30), one may get

$$
\tilde{F}\left(\omega, t_{0}\right)=|\omega|^{\frac{1}{2}} \int_{-\infty}^{\infty} f(t) e^{-i \omega t} g\left(\omega\left(t-t_{0}\right)\right) d t
$$


On dividing Equation (1.32) by $e^{-i \omega t_{0}}$ and later replacing $\omega$ with $\frac{1}{a}$ (thus, scale becomes inverse of frequency), one may obtain the following:

$$
\frac{\tilde{F}\left(\omega, t_{0}\right)}{e^{-i t_{0} / a}}=\frac{1}{|\alpha|^{\frac{1}{2}}} \int_{-\infty}^{\infty} f(t) e^{-i\left(t-t_{0}\right) / a} g \frac{t-t_{0}}{a} d t
$$

From the last equation, the wavelet function may be identified as $(t)=e^{i t} g(t)$, which represents the mother wavelet. On using translating and dilating parameters ( $b$ and $a$, respectively) in the mother wavelet, a series of other wavelets (of the same family) may be obtained. The term $b$ precisely denotes the time position around which the wavelet basis function is centrally placed, and the term $a$ represents the decomposition level (scale) of the wavelet transform. The Fourier transform of the mother wavelet basis function is given by

$$
{ }_{\hat{a}, b}\left(\omega, t_{0}\right)=e^{i b \omega} \frac{1}{\hat{a}}(\omega)
$$

With these definitions, Equation (1.33) takes the following form, which is the definition of the continuous wavelet transform [4]:

$$
{ }_{a}, b\left(\omega, t_{0}\right)=\frac{1}{|a|^{1 / 2}} \int_{-\infty}^{\infty} f(t) \frac{t-t_{0}}{a} d t
$$

For the wavelet transform to contain complete information of $\ddot{x}_{g}(t)$, the normalization constant has to be of finite value $\left(C_{\psi}<\infty\right)$, which obviously means that $\int_{-\infty}^{\infty} \psi_{a, b}(t) d t=0$. This, in turn, emphasizes that the wavelet function has finite energy and hence must vanish. This is called the admissibility condition of wavelet transform.

The discrete wavelet transform helps in analysing discrete signals or sampling continuous signals. The scales and time locations of the wavelet functions in case of discrete wavelet transform cannot take arbitrary values because the discretization rule obeyed by the specific wavelet scheme specifies the scales and time positions. The wavelet functions at various scales have variable size (but constant shape). The wavelet family in such a case is defined as

$$
{ }_{j, k}(t)=a_{0}^{j / 2}\left(a_{0}^{j}(t-b)\right)
$$


The dyadic wavelet scheme is another useful discretization scheme. In this case, the scale parameter is sampled on a dyadic grid. The corresponding discrete wavelet transform is defined as follows:

$$
\hat{a}_{, b}\left(a_{j}, b_{i}\right)=2^{j / 2} \int_{-\infty}^{\infty} f(t)\left(2^{j} t-k\right) d t
$$

In the above equation for discrete wavelet transform, $a_{j}=2^{j}$ and $b_{i}=k 2^{i}$. The integral in Equation (1.37) is performed in discrete format using the pyramid algorithm or fast wavelet algorithm [5, 6]. Usually, discrete wavelet transform (DWT) provides an efficient representation of a stochastic function compared to the representation by continuous wavelet transform (CWT) because DWT uses fewer scales and time positions to decompose a signal. The CWT helps in identification of singularities in the signals that might be ignored by a discrete grid. The computation of integrals for CWT and DWT is, however, not discussed here.

\section{I.5.2 Vanishing moments}

Another important property of the wavelet transform is the number of vanishing moments that represent the regularity of the wavelet functions and ability of a wavelet transform to capture localized information. A wavelet $\Psi(t)$ has $M$ vanishing moments if the following condition is satisfied:

$$
\int_{-\infty}^{\infty} t^{m}(t) d t=0 ; \quad m=0,1,2, \ldots, M-1
$$

The number of vanishing moments is directly related to the regularity of the wavelet. A more regular wavelet has a greater number of vanishing moments. The regularity of a function is expressed by the order of differentiability. For most of the wavelet functions, such as the Daubechies family of wavelets, the number of vanishing moments and the degree of differentiability are known. A wavelet of zero mean has at least one vanishing moment. A less regular wavelet function is suited more for the analysis of nonstationary data, whereas a more regular or smoother wavelet function is suitable for the analysis of stationary data. The localization property is another important feature of wavelets that helps capture the localized effects in the time domain as well as the frequency domain. Localization and regularity are inversely related to each other. Thus, depending on the 
specific characters of a signal to be analysed, preference between regularity and localization has to be made.

\section{I.5.3 Uncertainty principle}

The resolution in the time-frequency plane is limited by the uncertainty principle. The uncertainty principle states that contraction in the time domain dilates the function in the frequency domain. This means any effort to improve time localization will come at the cost of poor frequency resolution. This is as per the Heisenberg uncertainty principle, which is expressed as

$$
\sigma_{t} \sigma_{p} \geq \frac{1}{2}
$$

This signifies that a reduction in the value of the standard deviation in time (thereby improving time resolution) must increase the value of the standard deviation in frequency (thus reducing frequency resolution). Thus, the user has to decide the priority of one over the other and select the wavelet functions accordingly. The Shannon wavelet provides a good frequency resolution and is defined by

$$
(t)=\frac{\sin \{2 \pi(t-0.5)\}}{2 \pi(t-0.5)}-\frac{\sin \{\pi\}}{\pi(t-0.5)}
$$

The Shannon wavelet function is infinitely differentiable, and it has an infinite number of vanishing moments. The Fourier transform of the Shannon wavelet results in the following:

$$
(\hat{\omega})=e^{-i \frac{\omega}{2}} ; \quad \omega \in[-2 \pi,-\pi] \cup[\pi, 2 \pi]
$$

However, Shannon wavelets have poorer time resolution. Daubechies wavelets also offer good frequency resolution with increasing order. On the other hand, harmonic wavelets may give good time and frequency resolution together (to some extent), depending on suitable values of the parameters of harmonic wavelets. An example of a harmonic wavelet and its Fourier transform is given below [9]:

$$
f(t)=\int_{-\infty}^{\infty} \hat{F}(\omega) e^{i \omega t} d \omega=\frac{e^{i 4 \pi \omega t}-e^{i 2 \pi \omega t}}{i 2 \pi t}
$$


On taking Fourier transform, the frequency domain representation of the harmonic wavelet function may be written as

$$
\begin{aligned}
\hat{F}(\omega) & =\frac{1}{2 \pi} ; & & 2 \pi \leq \omega \leq 4 \pi \\
& =0 ; & & \text { otherwise }
\end{aligned}
$$

Though the harmonic wavelet is a complex function with real and imaginary components as even function and odd function, respectively, it has practically a finite support (hence, it contains finite energy).

Several authors in the last few decades have written quite a good number of books on wavelets [9-11]. However, a book on application of wavelets, especially with reference to civil engineering problems, is not really known to exist. This book may be viewed as a sincere effort to fill in that gap. 


\section{References}

1. Haar, A. (1910). Zur theorie der orthogonalen Funktionensysteme (Erste Mitteilung). Math. Ann., 69, 331-371.

2. Stromberg, J.O. (1982). A modified Franklin system and higher order spline systems on Rn as unconditional bases for Hardy spaces. In W. Beckner et al. (eds.), Proceedings of Conference in Honor of A. Zygmund, Vol. II, Wadsworth Mathematics Series, pp. 475-493.

3. Meyer, Y. (1992). Wavelets and operators. Cambridge University Press, New York.

4. Grossmann, A., and Morlet, J. (1984). Decomposition of Hardy functions into square integrable wavelets of constant shape. SIAM J. Math. Anal., 15(4), 723-736.

5. Mallat, S. (1989). Multiresolution approximation and wavelets. Trans. Am. Math. Soc., 315, 69-88.

6. Mallat, S. (1989). A theory for multi-resolution signal decomposition the wavelet representation. IEEE Pattern Anal. Machine Intell., 11(7), 674-693.

7. Daubechies, I. (1996). Where do wavelets come from? Proc. IEEE, 84(4), 510-513.

8. Daubechies, I. (1992). Ten lectures on wavelets. Society for Industrial Applied Mathematics, Philadelphia, PA, 1992.

9. Newland, D.E. (1993). An introduction to random vibrations, spectral and wavelet analysis. Longman, Harlow, Essex, UK.

10. Kaiser, G. (2010). A friendly guide to wavelets. Modern Birkhäuser Classics, Birkhäuser, New York.

11. Chui, C.K. (1992). An introduction to wavelets. Academic Press, San Diego, CA.

12. Basu, B., and Gupta, V.K. (1998). Seismic response of SDOF systems by wavelet modeling of nonstationary processes. ASCE J. Eng. Mech., 124(10), 1142-1150.

13. Alkemade, J.A.H. (1993). The finite wavelet transform with an application to seismic processing. In T.H. Koornwinder (ed.), Wavelets: An elementary treatment of theory and applications. World Scientific Co. Pte. Ltd., Hackensack, NJ, pp. 183-208.

14. Vanmarcke, E.H. (1975). On the distribution of the first-passage time for normal stationary random processes. ASME J. Appl. Mech., 42, 215-220.

15. Gupta, V.K., and Trifunac, M.D. (1993). A note on the effects of ground rocking on the response of buildings during 1989 Loma Prieta earthquake. Earthquake Eng. Eng. Vibration, 13(2), 12-28. 
16. Basu, B., and Gupta, V.K. (1997). Nonstationary seismic response of MDOF systems by wavelet transform. Earthquake Eng. Struct. Dyn., 6, 1243-1258.

17. Basu, B., and Gupta, V.K. (2000). Stochastic seismic response of single-degreeof freedom systems through wavelets. Eng. Struct., 22, 1714-1722.

18. Wong, H.L., and Trifunac, M.D. (1979). Generation of artificial strong motion accelerograms. Earthquake Eng. Struct. Dyn., 7, 509-527.

19. Lee, V.W., and Trifunac, M.D. (1985). Torsional accelerograms. Soil Dyn. Earthquake Eng., 4(3), 132-139.

20. Lee, V.W., and Trifunac, M.D. (1987). Rocking strong earthquake accelerograms. Soil Dyn. Earthquake Eng., 6(2), 85-89.

21. Lee, V.W., and Trifunac M.D. (1989). A note on filtering strong motion accelerograms to produce response spectra of specified shape and amplitude. Eur. Earthquake Eng., 111 (2), 38-45.

22. Trifunac, M.D. (1990). Curvograms of strong ground motions. ASCE J. Eng. Mech., 116(6), 1426-1432.

23. Housner, G.W. (1963). The dynamic behaviour of water tanks. Bull. Seismol. Soc. Am., 53(2), 381-387.

24. Haroun, M.A. (1983). Vibration studies and tests of liquid storage tanks. Earthquake Eng. Struct. Dyn., 11(2), 179-206.

25. Peek, R. (1988). Analysis of unanchored liquid storage tanks under lateral loads. Earthquake Eng. Struct. Dyn., 16(7), 1087-1100.

26. Peek, R., and Jennings, P.C. (1988). Simplified analysis of unanchored tanks. Earthquake Eng. Struct. Dyn., 16(7), 1073-1085.

27. Veletsos, A.S., and Tang, Y. (1990). Soil-structure interaction effects for laterally excited liquid storage tanks. Earthquake Eng. Struct. Dyn., 19, 473-496.

28. Chatterjee, P., and Basu, B. (2001). Non-stationary seismic response of tanks with soil interaction by wavelets, Earthquake Eng. Struct. Dyn., 30, 1419-1437.

29. Malhotra, P.K. (1997). Seismic response of soil-supported unanchored liquid storage tanks. ASCE J. Struct. Eng., 123(4), 440-450.

30. Veletsos, A.S., and Tang, Y. (1986). Dynamics of vertically excited liquid storage tanks. ASCE J. Struct. Div., 112, 1228-1246.

31. Chatterjee, P., and Basu, B. (2004). Wavelet-based non-stationary seismic rocking response of flexibly supported tanks. Earthquake Eng. Struct. Dyn., 33, 157-181.

32. Veletsos, A.S., and Verbic, B. (1973). Vibrations of visco-elastic foundations. Earthquake Eng. Struct. Dyn., 2, 87-102.

33. Wolf, J.P. (1988). Soil-structure interaction analysis in time domain. PrenticeHall, Englewood Cliffs, NJ.

34. Kondner, R.L. (1963). Hyperbolic stress-strain response: cohesive soils. ASCE J. Soil Mech. Found Div., 89, 115-143.

35. Lambe, T.W., and Whitman, R.V. (1979). Soil mechanics, SI version. Massachusetts Institute of Technology, Cambridge, MA.

36. Das, B.M., and Seeley, G.R. (1975). Load-displacement relationship for vertical anchor plates. ASCE J. Geotech. Eng., 101(GT7), 711-715.

37. Chang, C.Y., Power, M.S., Tang, Y.K., and Mok, C.M. (1989). Evidence of nonlinear soil response during a moderate earthquake. In Proceedings of 12th International Conference on Soil Mechanical and Foundation Engineering, Vol. 3, pp. 1927-1930. 
38. Roberts, J.B., and Spanos, P.D. (1989). Random vibration and statistical linearization. Wiley, New York.

39. Nayfeh, A.H., and Mook, D.T. (1979). Nonlinear oscillations. John Wiley, New York.

40. Basu, B., and Gupta, V.K. (1999). Wavelet based analysis of the nonstationary response of a slipping foundation. J. Sound Vib., 222(4), 547-563.

41. Ghanem, R., and Romeo, F. (1999). A wavelet based approach for the identification of linear time-varying dynamical systems. J. Sound Vibration, 234(4), 555-576.

42. Ghanem, R., and Romeo, F. (2001). A wavelet based approach for model and parameter identification of nonlinear systems. Int. J. Nonlinear Mech., 36(5), 835-859.

43. Chatterjee, P., and Basu, B. (2006). Nonstationary seismic response of a tank on bilinear hysteretic soil using wavelet transform. Probabilistic Eng. Mech., 21, 54-63.

44. Chatterjee, P., Obrien, E., Li, Y., and Gonzalez, A. (2006). Wavelet domain analysis for identification of vehicle axles from bridge measurements. Comput. Struct., 84, 1792-1801

45. Basu, B. (2005). Identification of stiffness degradation in structures using wavelet analysis. Constr. Building Mater., 19, 713-721.

46. Livaoglu, R. (2008). Investigation of seismic behavior of fluid-rectangular tank-soil/foundation systems in frequency domain. Soil Dyn. Earthquake Eng., 28(2), 132-146.

47. Tam, L. (2008). Seismic response of liquid storage tanks incorporating soil structure interaction. ASCE J. Geotech. Geoenviron. Eng., 134(12), 1804-1814.

48. Ding, W., and Sun, Z. (2014). Wavelet-based response of computation for baseisolated structure under seismic excitation. J. Appl. Math. Phys., 2, 163-169.

49. Ovanesova, A.V., and Suárez, L.E. (2004). Applications of wavelet transform to damage detection in frame structures. Eng. Struct., 26, 39-49. 
